Revista Iberoamericana, Vol. LXXVIII, Núms. 238-239, Enero-Junio 2012, 425-439

\title{
EL BOOM DE LA CIENCIA-FICCIÓN ARGENTINA EN LA DÉCADA DEL OCHENTA
}

\author{
POR \\ Luis Pestarini \\ Biblioteca Nacional \\ Revista Cuásar
}

Hace poco más de medio siglo, el 7 de mayo de 1959, en el Senate House de Cambridge, C. P. Snow ofreció una conferencia sobre uno de los tópicos que marcaron el siglo xx: la ruptura entre las dos culturas occidentales, las humanidades y las ciencias. Snow sabía de qué hablaba: era físico y novelista y conocía ambos mundos a través de su propia experiencia. La conferencia se convirtió poco después en un texto clásico, Las dos culturas y la revolución científica.

Snow argumentaba que había una profunda incomprensión entre ambas culturas, lo que se traducía en gran parte de los conflictos modernos, y que el origen de esta incomprensión estaba en una educación deficiente, en continua decadencia. Participante de tertulias de novelistas y de científicos por separado, solía hacer notar que los científicos no habían leído a Dickens para burla de los literatos y, por otro lado, los novelistas desconocían de qué trataba la Segunda Ley de la Termodinámica; ambos saberes eran despreciados por su contraparte, pero importantes por igual.

Lo que Snow no sabía y tal vez nunca intuyó es que hay un territorio donde estas dos culturas cohabitan: la ciencia-ficción. Este género, de origen literario, puede pensarse como un espacio donde ambas formas del pensamiento se entrelazan para producir una obra única. Si bien ésta es una simplificación algo extrema, ayuda a comprender por qué es un género en el margen de la literatura, con escasa atención de la academia y que espanta a los escritores consagrados, aún cuando algunos de sus textos estén ambientados en el futuro o en universos paralelos (dos de los ambientes preeminentes de este género). Algunos escritores consagrados como Philip Roth y Margaret Atwood son ejemplos de estos escritores que hacen grandes esfuerzos para evitar que sus novelas sean rotuladas como ciencia-ficción.

Ahora bien, la ciencia-ficción se ha desarrollado esencialmente en los llamados países desarrollados, en especial en Gran Bretaña, Estados Unidos y Francia, pero ejemplos de este género pueden encontrarse en casi todas las literaturas nacionales, las de América Latina no son la excepción. Miguel Ángel Fernández Delgado, el más consistente historiador de la ciencia-ficción mexicana, localizó un primer manuscrito 
redactado en 1775 por el fraile franciscano Manuel Antonio de Rivas, mientras que en Argentina el antecedente más lejano conocido se remonta a un mes antes de la declaración de la Independencia, en junio de 1816, cuando un periódico publicó de manera anónima una sátira de costumbres denominada "Delirio".

La segunda mitad del siglo xix y la primera del xx muestran la cada vez más frecuente aparición de textos que pueden ser encuadrados dentro de la ciencia-ficción, en particular en Argentina, Brasil, México y Chile. ${ }^{1}$ De la literatura del primero de estos cuatro países nos vamos a ocupar en este breve texto.

Aunque hemos señalado que el primer texto de ciencia-ficción publicado en territorio argentino -entonces Provincias Unidas del Río de la Plata- es de 1816, al que se suman ejemplos posteriores, se suele considerar a algunas obras de Eduardo Ladislao Holmberg (1852-1937) como las fundacionales del género, en particular Viaje maravilloso del señor Nic-Nac en el que se refieren las prodigiosas aventuras de este señor y se dan a conocer las instituciones, costumbres y preocupaciones de un mundo desconocido (1875), “Horacio Kalibang o los autómatas” (1879) y “Filigranas de cera” (1884): Los relatos de ciencia-ficción continuaron publicándose irregularmente durante el resto del siglo xix y la primera mitad del xx, algunos firmados por nombres reconocidos como Lugones, Hugo Wast, Manuel Ugarte o Arturo Cancela, pero no se configuró como género y raramente se hacía referencia a ellos como antecedente literario, una característica común en la ciencia-ficción.

La situación cambió con la publicación de la novela La invención de Morel (1940), de Adolfo Bioy Casares. Esta historia sobre la inmortalidad tiene como referencia a Wells y, en particular, a La isla del doctor Moreau. Borges, en su ya famoso prólogo, la llama una obra de "imaginación razonada”, una expresión simétrica a ciencia-ficción (ficción=imaginación y razonada=ciencia), lo que ha llevado a algún crítico (citado en Brown 475) a señalar que Borges rechazaba la denominación “ciencia-ficción” y al género mismo, sin tener en cuenta que esta denominación se usó por primera vez en español en 1955. Considerada muchas veces como la mejor novela argentina de ciencia-ficción, es el punto más alto en la obra de Bioy Casares y de su intensa relación con el género que comenzó con algunas de sus primerizas e irrelevantes obras y culminó con su última narración publicada en vida, De un mundo a otro (1998), también un relato wellsiano.

Estas obras dispersas no alcanzan a ser más que ejemplos aislados de textos de ciencia-ficción que no definen aún un espacio propio dentro de la literatura argentina. Este espacio comienza a tomar forma en los cincuenta con dos hitos: la publicación de la revista Más allá, con cuarentiocho ediciones mensuales entre 1953 y 1957, y la

\footnotetext{
1 Véase “A Chronology of Latin-American Science Fiction, 1775-2005”, de Molina-Gavilán y otros, para profundizar. Este trabajo merece ser actualizado ya que, por ejemplo, sólo en el área de Argentina hay al menos unos veinte títulos localizados con posterioridad a la publicación.
}

\footnotetext{
Revista Iberoamericana, Vol. LXXVIII, Núms. 238-239, Enero-Junio 2012, 425-439
ISSN 0034-9631 (Impreso)
} 
aparición de la editorial Minotauro, fundada por Francisco “Paco” Porrúa, en 1955, con la presentación de clásicos del género en cuidadas presentaciones y con prólogos de Jorge Luis Borges y Marcos Victorica, en una operación de legitimación de la cienciaficción ante un lector “culto”. Más allá no tenía esas pretensiones; estaba dirigida a un lector menos exigente, con artículos de divulgación científica y relatos traducidos de las revistas estadounidenses Astounding y Galaxy, más ocasionales aportes locales. Pero lo que por primera vez se conformó fue un importante grupo de seguidores, una auténtica comunidad que se manifestó abundantemente en la sección de correo, y que puede ser considerado como el primer fandom argentino, que después no tuvo continuidad.

Conviene hacer un apartado para explicar una característica singular que tiene la comunidad de lectores de ciencia-ficción a lo ancho del mundo: tienden a ser gregarios, a reunirse, organizar actividades, grupos de lecturas y producir sus propias publicaciones denominadas fanzines. El fandom (contracción de Fan Kingdom, el reino de los fanáticos) es un fenómeno que se originó en los Estados Unidos a comienzos de los treinta, que en Argentina tuvo sus primeras manifestaciones en torno a Más allá y que no puede escindirse de la producción literaria de ciencia-ficción si se la analiza como género.

Pablo Capanna (44-56) estableció períodos del desarrollo de la ciencia-ficción argentina: Implantación (1953-1955), Consolidación y crecimiento (1956-1965), Expansión (1966-1970), Repliegue (1971-1975), Segunda expansión (1976 en adelante). Vista un cuarto de siglo después de pensada, esta periodización puede simplificarse un poco. La consolidación y el crecimiento podrían extenderse hasta 1970, con particular énfasis en los últimos años de la década en la que aparecen la primera encarnación de la revista Minotauro (diez números entre 1964 y 1968) cuatro libros de autores argentinos publicados por la misma editorial y varias antologías. Curiosamente, aunque a la distancia se reconocen como textos menores, casi clichés de la tradición anglosajona de la ciencia-ficción, estos libros son los que parecen haber llamado más la atención de la crítica, en desmedro de otros más valiosos publicados con posterioridad. ${ }^{2}$

No parece haber una diferencia sustancial en la cantidad de publicaciones en las dos etapas en que Capanna divide la década del setenta, salvo por la aparición de Los universos vislumbrados (1978), una antología con una visión histórica de la cienciaficción argentina que incluye un estudio preliminar de Elvio E. Gandolfo sobre el género en la Argentina, el primer trabajo de este tipo, y por la aparición de cuatro revistas de ciencia-ficción que en total publicaron diez números -La revista de ciencia-ficción

2 Cuatro de los cinco relatos de autores argentinos seleccionados para Cosmos Latinos: An Anthology of Science Fiction from Latin America and Spain (Andrea L. Bell y Yolanda Molina-Galván, eds.), pertenecen a este período. Yolanda Molina-Gavilán, en Ciencia ficción en español, cuando analiza la ciencia-ficción argentina se detiene especialmente en obras del período. Aunque más razonable por la fecha de publicación (1980), los seis relatos argentinos seleccionados por el belga Bernard Goorden para Lo mejor de la ciencia-ficción latinoamericana pertenecen a aquellos años.

Revista Iberoamericana, Vol. LXXVIII, Núms. 238-239, Enero-Junio 2012, $425-439$
ISSN 0034-9631 (Impreso) 
y fantasía, Entropía, Suplemento de Humor y Ciencia Ficción y El Péndulo-, todas dirigidas por Marcial Souto, antecedente directo del boom de la ciencia-ficción en los '80, período del que intentaremos ofrecer una cartografía a lo largo de este artículo.

El rasgo principal de la década del ochenta es que se publica más literatura de ciencia-ficción que en toda la historia previa del género en la Argentina, en particular a través de las publicaciones periódicas especializadas, y se conforma el fandom donde concurren escritores, lectores, editores y traductores. Los noventa marcaron una etapa de aridez, con un fandom retraído y escasas ediciones de libros y revistas, hasta que la primera década del siglo xxi exhibió un nuevo florecimiento, en gran medida por la proliferación de sitios en Internet dedicados al género.

Para comprender el boom de la ciencia-ficción argentina en aquella década es necesario exponer el contexto que facilitó este auge. En primer lugar, hacia 1980 la censura en los medios y la represión de toda actividad no autorizada por la dictadura militar comenzaban a mostrar importantes grietas. En una de ellas se alojó una publicación independiente que a un par de años de su fundación se convirtió en abanderada de la lucha por el regreso a un régimen democrático y en una muy ácida burla del gobierno militar. La contribución de la revista quincenal $\operatorname{Hum}{ }^{\circledR}$, creada y dirigida por Andrés Cascioli, al desgaste y retiro del gobierno militar no puede ser subestimada: sus posturas fueron cada vez más críticas, provocando persecución e incluso el secuestro de una edición antes de su distribución, pero llegó a vender trecientos treinta mil ejemplares quincenales, una cifra nunca alcanzada por una revista de esas características.

A mediados de los setenta, Marcial Souto y el uruguayo Jaime Poniachik le propusieron a Cascioli, que entonces dirigía la publicación humorística Chaupinela, hacer una revista de literatura fantástica y ciencia-ficción. El proyecto se puso en marcha, pero antes de la aparición del primer número sobrevino una aguda crisis económica que lo congeló. Souto había nacido en La Coruña en 1947, era autodidacta y sus intereses lo llevaron primero a Montevideo, donde publicó una breve colección de libros, luego a Estados Unidos, donde conoció a los principales escritores de ciencia-ficción. No se desalentaba con facilidad. Logró llamar la atención de Ediciones Orión que, bajo su dirección, publicó tres números de La revista de ciencia-ficción y fantasía, y luego fundó la Editorial Entropía (dos libros y un número de la revista homónima). A fines de los setenta se acercó nuevamente a Cascioli y consiguió que se volviera a interesar en el viejo proyecto. Ediciones de la Urraca, cuya revista Hum ${ }^{\circledR}$ todavía no había alcanzado la cima de la popularidad, probó el mercado sacando dos números de una publicación llamada Suplementos de Hum ${ }^{\circledR}$ y Ciencia-Ficción, en junio y julio de 1979, reproduciendo el formato de la revista $\operatorname{Hum}{ }^{\circledR}(28$ x $20 \mathrm{~cm})$, con cuentos, historietas y notas sobre el género. En la última página del segundo suplemento se anunció una nueva publicación: El Péndulo: entre la ficción y la realidad, revista de Literatura Ilustrada, humor e historietas.

\footnotetext{
(3) Revista Iberoamericana, Vol. LXXVIII, Núms. 238-239, Enero-Junio 2012, 425-439 ISSN 0034-9631 (Impreso) 
El Péndulo no cambió mucho con respecto al Suplemento, que se había referenciado en la revista Hum ${ }^{\circledR}$ desde el título. El Péndulo, en esta primera etapa, alcanzó a publicar cuatro números antes de ser suspendida, todos en 1979. En ellos tienen poco espacio los autores argentinos (básicamente hay crítica y ensayo a cargo de Pablo Capanna y Elvio E. Gandolfo), pero permiten reconocer la existencia de un lector que puede sostener publicaciones de este tipo, en la estela de la cada vez más exitosa Hum ${ }^{\circledR}$. Pero las tensiones entre los distintos contenidos, las historietas y el humor, por un lado, y la crítica y la narrativa literaria corta, por el otro, terminaron congelando la publicación durante 1980 para que, un año más tarde, se produjera la división de la revista en dos. Según sus contenidos la revista de dividió por un lado en Superhum ${ }^{\circledR}$, que mantuvo el formato, dedicada a la historieta, dirigida por Juan Sasturain, y que con los años daría paso a la mítica Fierro, y un segundo ciclo de El Péndulo, con formato más cercano al libro (22,5 x $17 \mathrm{~cm})$ y ya completamente bajo la dirección de Marcial Souto, dedicado a la narrativa literaria del género.

En este nuevo ciclo se publicaron diez números entre mayo de 1981 y noviembre de 1982. La producción gráfica (ilustraciones, diagramación y tapas) fueron de una calidad y originalidad nunca alcanzadas por una publicación del género, y la selección de relatos y artículos, más las cuidadas traducciones, no se quedaban atrás. Pero el aporte de los autores argentinos fue muy escaso en el rubro de relatos (diez en otras tantas ediciones), aunque el espacio para artículos y ensayos fue más amplio, especialmente los escritos por los ya mencionados Capanna y Gandolfo.

La tercera encarnación de El Péndulo, cinco ediciones con numeración continua con la anterior -es decir que llega al número 15- se da entre septiembre de 1986 y mayo de 1987. A un cierto aggiornamiento del diseño se suma un mayor espacio para los relatos de autores argentinos que alcanzaron la quincena. La última encarnación de la publicación se dio entre 1991 y 1992, cuando aparecieron dos ediciones de El Péndulo Libro, con una estética mucho más sencilla y un amplio espacio para los autores argentinos.

El impacto de El Péndulo en los lectores fue grande y perdura aún hoy. La refinada combinación de literatura fantástica con una gráfica innovadora y dinámica, alejada de los monótonos diseños de las revistas de literatura, la promoción desde otras publicaciones de la editorial dirigidas a un lector "culto", y la deliberada omisión de la expresión ciencia-ficción en la tapa, le permitieron alcanzar un amplio público, algo desconocido para las revistas de este tipo desde la época de Más allá.

La labor de promoción de lo fantástico que llevó adelante Marcial Souto en los ochenta fue enorme y no hay forma de medirla, pero de ninguna forma concluye con su dirección de El Péndulo: en abril de 1983 apareció el primer número de la revista Minotauro, también bajo su dirección, con distribución únicamente en librerías. La revista estaba un poco más inclinada hacia el fantástico surrealista, aunque de ningún modo estuvo ausente la ciencia-ficción, pero más allá de esto y las características de un

\footnotetext{
Revista Iberoamericana, Vol. LXXVIII, Núms. 238-239, Enero-Junio 2012, 425-439 ISSN 0034-9631 (Impreso) 
diseño más contenido, se la advierte como una revista hermana. Tenía al mismo equipo de colaboradores (Capanna, Gardini, Gandolfo, Aníbal Vinelli y Elvira Ibarguen) y se repitieron los escritores fetiche: Mario Levrero, Cordwainer Smith, Philip Dick, Angélica Gorodischer y Ballard, con pocas variaciones. Publicó once números hasta marzo de 1986. Además, la décima edición estaba dedicada íntegramente a la literatura argentina. Acompañando a estas verdaderas antologías, la editorial Minotauro publicó una serie de pequeños volúmenes de autores argentinos (y del uruguayo Levrero) de una literatura a veces inclasificable, otras fantástica y en ocasiones de ciencia-ficción. Entre estos títulos merecen mencionarse Mi cerebro animal y Juegos malabares, de Carlos Gardini; Kalpa Imperial, de Angélica Gorodischer; El fondo del pozo, de Eduardo Abel Giménez; Las escamas del señor Crisolaras, de Rogelio Ramos Signes; y Cuerpos descartables, de Sergio Gaut vel Hartman.

Souto publicó en este período dos libros de cuentos breves, mayormente fantásticos, y realizó una extensa labor como traductor, pero es indudable que su marca sobre la ciencia-ficción argentina la dejó por su labor. Fue el continuador natural de Francisco Porrúa en su labor en Minotauro desde los cincuenta, con un rigor en las traducciones y presentaciones de los textos como no se había visto antes. Los relatos de autores argentinos que publicó, en términos generales, parecen herederos de la tradición fantástica argentina más que de la ciencia-ficción anglosajona, aún en los casos en que pueden ser encuadrados como ciencia-ficción; además, difundió ampliamente la obra única de Mario Levrero. Tal vez se pueda afirmar que su paleta de autores no fue muy amplia y que tuvo una clara predilección por los autores anglosajones que dieron sus mejores obras en los sesenta y principios de los setenta, pero sus méritos fueron reconocidos ampliamente, como se desprende de las palabras del crítico e historiador sueco Sam J. Lundwall: “La mejor revista norteamericana, The Magazine of Fantasy \& Science Fiction, es una de las tres mejores del mundo (las otras dos son la argentina El Péndulo, sin duda la mejor revista de ciencia-ficción en contenido, presentación y diseño jamás publicada, y la húngara Galaktika)” (73).

En el correo de lectores de la sexta edición de El Péndulo, en enero de 1982, había una carta firmada por Sergio Gaut vel Hartman convocando a los aficionados y escritores de ciencia-ficción y proponiendo un encuentro del “fandom nacional”. El 5 de febrero se organizó la primera reunión y se puede hablar de esta fecha como el inicio formal de las actividades del fandom argentino que continúa hasta nuestros días. En abril apareció el primer Boletín (de apenas una hoja plegada) del Círculo Argentino de Ciencia-Ficción y Fantasía (Cacyf), que continuaría con sus actividades hasta fines de los noventa, publicando más de setenta números del Boletín (algunos de casi cien páginas). El Cacyf fue un espacio que fomentó las actividades vinculadas con la ciencia-ficción y la fantasía, con reuniones semanales donde llegaron a juntarse ochenta personas, actividades como conferencias o ciclos de cine. También permitió organizar concursos

\footnotetext{
Revista Iberoamericana, Vol. LXXVIII, Núms. 238-239, Enero-Junio 2012, 425-439 ISSN 0034-9631 (Impreso) ISSN 2154-4794 (Electrónico)
} 
para relatos inéditos y otorgó durante más de una década los premios Más Allá a la producción de ciencia-ficción y fantasía argentina, en distintas categorías (novela, ensayo, cuento, revista, etc.), elegidos por el voto de los asociados. El Boletín reviste valor no sólo porque incluye una crónica de las actividades que se llevaban adelante, sino también porque anualmente publicaba una extensa lista de todos los trabajos de ciencia-ficción y fantasía publicados, como guía para las nominaciones para el premio Más Allá, lista que si bien no es una bibliografía, constituye una fuente fiable de información.

Esta efervescencia relacionada con un género literario no sólo estaba vinculada con la actitud gregaria de los aficionados y por la publicación más o menos regular de dos revistas con grandes tiradas, sino también con las posibilidades que brindaba la agonía del régimen militar, de por sí reacio a cualquier dinámica que proviniera del mundo de la cultura, que permitió varios fenómenos paralelos, entre ellos, por ejemplo, Teatro Abierto. Las nacientes actividades del Cacyf se dieron en este marco.

El siguiente objetivo natural, una vez establecido el funcionamiento del Cacyf, fue el de tener una publicación literaria propia. Como se puede apreciar en los primeros números del Boletín, hubo un debate que fue saldado de la siguiente manera: el Cacyf continuaría con su Boletín, mientras que cualquier otra publicación sería editada por socios o grupos de socios de manera independiente a la asociación. Los diferentes programas literarios se hicieron evidentes con las apariciones de las primeras revistas o fanzines en 1983 y 1984.

Vale hacer un paréntesis para aclarar el término fanzine con que se denominaba a las publicaciones producidas de manera independiente. La expresión fanzine se emplea básicamente en el mundo anglosajón para denominar a las publicaciones que difunden las actividades, la historia y todo lo relacionado con el fandom vernáculo, pero no son revistas literarias. En Argentina -y en otros lugares de América Latina-, los fanzines replicaban el tipo de contenido de las revistas con distribución comercial (cuentos, traducciones, ensayos, crítica, etc.), con tiradas que iban de los cincuenta a los quinientos ejemplares, y con una calidad y presentación extremadamente variables, pero siempre fueron revistas literarias.

La primera publicación de este tipo realizada en el marco del Cacyf (hay algunos efímeros antecedentes en las décadas del sesenta y setenta) fue Sinergia, editada por Gaut vel Hartman, quien sin ninguna duda puede ser considerado una figura decisiva del período como editor, promotor de actividades culturales y escritor, actividades que aún hoy continúa llevando adelante. Sinergia publicó el primer número en el verano de 1983 y culminó su andadura con el doceavo número, fechado en el otoño de 1987, ya con una presentación que podía competir con las publicaciones con distribución comercial. Aunque publicaba relatos traducidos de otros idiomas (inglés, ruso, francés), su fuerte siempre fue la narrativa en español. No fue frecuente en esta vertiente una ciencia-ficción de corte clásico, sino una más bien experimental, a veces con toques

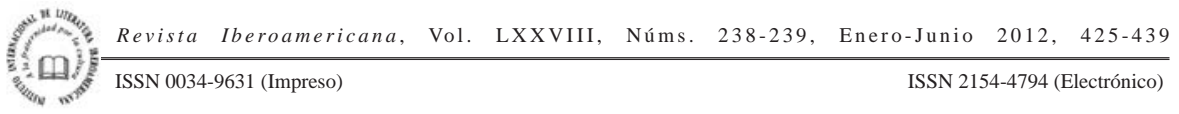


de absurdo, cercana a la new wave inglesa de los sesenta. Pasaron por sus páginas los autores más destacados del período: Gorodischer, Gardini, Gandolfo, Levrero, Eduardo Abel Giménez y el mismo editor, muchas veces bajo seudónimo. Puede subrayarse, entre todos los relatos publicados, uno que podría tomarse como paradigma de la ciencia-ficción argentina: "Planetas de papel”, de Claudia De Bella (más conocida como traductora). Con una lograda ambientación futurista con tintes argentinos y un lenguaje acorde, la historia narra los abusos que ejerce sobre sus empleados argentinos una empresa dedicada a la colonización de planetas.

El siguiente título en aparecer fue Nuevomundo, en septiembre/octubre de 1983. Con una presentación más prolija que la que había tenido inicialmente Sinergia -que por entonces ya estaba publicando su tercer número, con un diseño muy mejorado-, Nuevomundo fue editada por Daniel M.A. Croci, quien firmaba sus ficciones como Daniel Barbieri. La revista tenía una línea mucho más definida que sus colegas: en el editorial de su primer número, "Las dos maneras de escribir”, fustiga el relato experimental, subjetivo o poético, inspirado, según señala Croci, en la new wave de la ciencia-ficción inglesa, prefiriendo una ciencia-ficción accesible, realista, escrita por autores argentinos con ambientes y personajes argentinos. Y si bien no siempre los relatos cumplieron con esta premisa, a lo largo de las dieciséis ediciones publicadas entre 1983 y 1991 -las últimas dirigidas por Santiago Oviedo-, mantuvo una envidiable coherencia. En el cuarto número aparece un manifiesto donde el editor reflexiona sobre su postura de una literatura fantástica, incluyendo la ciencia-ficción, nacional y popular:

Los conflictos, esperanzas y temores de este tiempo han de reflejarse en una nueva literatura fantástica nacional. Por eso el escritor que quiera ser popular debe compenetrarse con nuestra realidad, hacerla parte de sí mismo y hacerse parte de ella antes de largarse a fantasear. La técnica literaria y la variedad de recursos narrativos son importantes, pero son artesanía, no arte. Mucho más importante es la capacidad imaginativa y aún más el talento de comunicar mensajes que llegue al pueblo lector, por ser el reflejo transfigurado, extrapolado, de lo que ese mismo pueblo siente y no puede decir.(Croci 40)

Croci (1951-2004), que nunca escapó a la discusión política desde su publicación y también desde otros medios, fue un personaje polémico pero coherente, que intentó abrir un camino para una forma de la ciencia-ficción que no careció de seguidores. Como Daniel Barbieri, publicó varios relatos que seguían prolijamente sus planteos.

La tercera publicación en aparecer fue Cuásar, editada por quien suscribe esta nota y por Mónica Nicastro; durante un breve período, entre los números veinte y veintitrés (1990/91), la dirección fue compartida con Juan Carlos Verrecchia y Verónica Figueirido y, desde entonces, está a cargo únicamente de Pestarini. Cuásar es la única publicación impresa que continúa editándose en la actualidad, la más antigua editada en español y la que se ha publicado a lo largo del mayor lapso de años, al borde del medio centenar

\footnotetext{
Revista Iberoamericana, Vol. LXXVIII, Núms. 238-239, Enero-Junio 2012, 425-439 ISSN 0034-9631 (Impreso) ISSN 2154-4794 (Electrónico)
} 
de ediciones. Su primer número salió en enero de 1984, cuyo editorial declaraba sus intenciones:

Cuásar tiene la pretensión -dentro de sus posibilidades- de ser un canal para que el escritor pueda dar a conocer sus creaciones, perfeccionar su lenguaje y asentar su estilo (y para leer las cosas del escritor consumado que no se editan en otro lado). Lo mismo vale para el traductor. Porque éste trabaja tanto como el autor, y el no reconocer esto trae como consecuencia las malas traducciones que nos bombardean todavía. (2)

De modesta presentación, la publicación procuraba estimular también una crítica más rigurosa que la habitual en las revistas de su tipo. El proyecto comenzó realmente a concretarse a partir del sexto número, con la inclusión de la novela corta "Un paseo con Gerónimo”, de Daniel Barbieri, relato escrito entre 1978 y 1982 que presenta a los desaparecidos como fantasmas que deambulan por la ciudad sin comprender qué les ha sucedido, por qué se han vuelto inmateriales, buscando desesperadamente acabar con esa no-existencia.

Hasta el fin de la década se publican una docena más de revistas, de muy variada calidad y presentación, que editan entre uno y quince números. La mayoría de ellas dedican sus páginas a los relatos de autores argentinos (y también muchos hispanoamericanos, pues el dinámico movimiento editorial atrajo la atención de los escritores en lengua española, a veces incluyendo algunos textos traducidos y algo de crítica). Entre estas publicaciones destacan Parsec, dirigida por Gaut vel Hartman, que publicó seis números mensuales en 1984 y tuvo distribución en kioscos, básicamente compuestos por relatos traducidos y que se destaca por la inclusión en tres entregas de la novela Un paseo por Camarjali, de Eduardo Abel Giménez. Otras publicaciones tenían tiradas muy reducidas, que rondaban los cien ejemplares, como Gurbo, que publicó once números mensuales entre 1985 y 1986, editada por Martín Salías y Tuqui, y que fue muy apreciada en el fandom, y Potencial, con cinco números entre 1986 y 1987, destinada a los autores noveles y editada por Gaut vel Hartman. También Vórtice (diez números entre 1986 y 1988), editada por Verrecchia y Figueirido, con su atildada mezcla de relatos argentinos y traducciones, tuvo su repercusión en ese universo de lectores.

No podemos dejar de mencionar que la década se cierra con la aparición de Axxón: ciencia-ficción en bits en 1989. Fue la primera revista en lengua española de cualquier disciplina en ser publicada en soporte informático (entonces disquetes de 5 1/4), con un programa para PC desarrollado especialmente por Eduardo J. Carletti (director de la publicación hasta el día de hoy) y Fernando Bonsembiante. Desde 2001 se publica directamente en Internet (axxon.com.ar) y ha superado las doscientas ediciones y los veinte millones de visitas. Su contenido siempre ha sido muy variado y abundante, pero poco está comprendido en el terreno del estudio de este artículo pues su primer número se distribuyó en septiembre de 1989.

\footnotetext{
Revista Iberoamericana, Vol. LXXVIII, Núms. 238-239, Enero-Junio 2012, 425-439 ISSN 0034-9631 (Impreso) ISSN 2154-4794 (Electrónico)
} 
Toda la actividad y las publicaciones que hemos mencionado tuvo lugar en la ciudad de Buenos Aires; pero hubo pequeños fandoms en otras ciudades como Rosario, Mar del Plata, Córdoba, Mendoza, Bahía Blanca y La Plata, pero fue sólo en la primera donde se produjeron publicaciones. Allí, Claudio Omar Noguerol editó quince números de Unicornio azul (luego El unicornio), entre 1984 y 1989, y otros quince de Supernova, entre 1985 y 1989. Estas publicaciones dieron amplio espacio a los autores santafesinos, pero también se pueden encontrar textos de los mismos escritores que aparecían una y otra vez en las revistas de Buenos Aires.

No es sencillo establecer en forma definitiva los motivos del alza y caída de las publicaciones de ciencia-ficción y fantasía en la Argentina de los ochenta. Es indudable que El Péndulo y, en menor medida, Minotauro (revista y colección de libros) sirvieron como polos magnéticos que atrajeron lectores y escritores, y que generaron un espacio para que aparecieran publicaciones de menor alcance. También el efervescente clima en todos los ámbitos culturales que significó el final de la dictadura militar y la nueva etapa democrática generaron espacios donde crecieron las iniciativas creativas, uno de ellos habitado por este género. Pero no deberían ser considerados menores otros factores que tendrían que ser explorados y puestos a prueba, como la habilitación para mirar el futuro, un mundo de tinieblas en la década anterior, que facilitó la ciencia-ficción, y la necesidad de reflexionar sobre la reciente Guerra de Malvinas desde un lugar que no fuera la crónica o el relato realista, algo que podría ser respaldado por la repetición del tema de la guerra y la violencia en los relatos del período.

Más sencillo puede ser determinar por qué este período llegó a su fin: la masiva desaparición de revistas en los últimos dos años de la década y la mínima aparición de títulos nuevos parece estar directamente vinculada con la crisis económica, hiperinflación inclusive, que sufrió Argentina. Las publicaciones con distribución comercial, pero también los fanzines, sintieron frontalmente las olas de la debacle. El modelo neoliberal instalado desde 1989 también funcionó como elemento desalentador para estas iniciativas, pero no deberían descuidarse otros posibles factores vinculados con lo creativo. Una parte significativa de la producción de algunos escritores prolíficos del período (Gaut vel Hartman, Gardini, Barbieri y Carletti, entre otros) había sido escrita previamente, en los setenta, y estaba esperando la aparición de los medios de publicación, pero esta cantera comenzó a agotarse y se comenzó a notar cierto empobrecimiento en la producción literaria de fines de la década y la falta de aparición de voces nuevas. Las revistas y publicaciones de esos años ofrecieron a los escritores un espacio donde dar a conocer sus obras, pero también fomentaron cierta endogamia literaria donde los editores eran también escritores, los mismos autores aparecían en todas las publicaciones, con el consiguiente empobrecimiento genético.

Con más de cincuenta libros y unas doscientas ediciones de publicaciones periódicas durante el período revisado, se torna muy difícil hacer un análisis más o menos abarcador

\footnotetext{
(3) Revista Iberoamericana, Vol. LXXVIII, Núms. 238-239, Enero-Junio 2012, 425-439 ISSN 0034-9631 (Impreso) ISSN 2154-4794 (Electrónico)
} 
sin dejar afuera obras y autores de relevancia, pero como ya señalamos, es un territorio poco explorado y es preferible apresurar unas líneas sobre los escritores que destacaron en la ciencia-ficción argentina de aquellos años que dejar vacante la cuestión. Sólo las obras de Ana María Shua, Angélica Gorodischer, Marcelo Cohen y, por supuesto, Bioy Casares han atraído la atención de la crítica, pero aún así, la parte de sus obras publicadas en estos años no es la más significativa en el corpus completo, salvo, tal vez, en el caso de Gorodischer. Darrell B. Lockhart en su repertorio Latin American Science Fiction Writers, tiene entradas sobre tres de los cuatro autores mencionados (omite a Cohen), y suma a Carlos Gardini, Gaut vel Hartman y Marcial Souto, una cosecha un tanto escasa para una obra de referencia de estas características, pero aún así es quien más se detiene en autores que produjeron una obra significativa de ciencia-ficción en los ochenta.

Antes de caracterizar brevemente la obra de algunos escritores relevantes, es oportuno destacar circunstancias que tal vez pasen desapercibidas. La primera de ellas es que la mayor parte de las revistas estaban dirigidas por escritores de narrativa, un fenómeno no tan usual, y algunas podrían considerarse incluso plataformas para difundir la obra propia, una situación nada ilegítima, por cierto. Pero como no había cercas autoimpuestas, un mismo autor podía aparecer en las publicaciones de mayor jerarquía (El Péndulo o Minotauro), pero también frecuentar publicaciones rústicas y de ediciones muy limitadas. Este factor, entre otros, generó un sentido de pertenencia a una comunidad que fortaleció las publicaciones y, a la vez, terminó retroalimentando al mismo fandom. Así, por ejemplo, hubo autores que llegaron a superar el medio centenar de cuentos publicados en el lapso de diez años, una cifra inimaginable en épocas anteriores.

Probablemente la obra más significativa y representativa del período, y de gran parte de las últimas tres décadas, dentro de la ciencia-ficción argentina, sea la de Carlos Gardini (1948). Aunque era conocido como traductor, actividad a la que sigue vinculado, como narrador se hizo conocer a partir de que su cuento "Primera línea”, que ganó en 1982 el Premio Círculo de Lectores, cuyo jurado integraban Borges y Donoso, entre otros. En el lapso de dieciocho meses publicó cuatro libros: Mi cerebro animal, Primera línea (1982, ambos de relatos), Juegos malabares (1983, novela articulada) y Sinfonía cero (1983, una novela corta y varios cuentos), pero debió esperar hasta 1993 para que su novela El libro de la tierra negra tuviera su primera edición. La variedad de registros de los relatos de Gardini es muy amplia, desde lo fantástico clásico hasta la ciencia-ficción futurista, pero es claramente reconocible su prosa firme y rigurosa. En particular en este período son frecuentes sus cuentos sobre la guerra y la violencia, que fueron vinculados muchas veces con reflexiones sobre la Guerra de Malvinas pero que, según el autor, fueron escritos en su mayoría antes. ${ }^{3}$ "Primera línea”, una historia

3 “Casi todos los cuentos fueron anteriores [a la Guerra de Malvinas], salvo "Primera línea”, que fue casi un corolario” (Gardini 121).

\footnotetext{
Revista Iberoamericana, Vol. LXXVIII, Núms. 238-239, Enero-Junio 2012, 425-439 ISSN 0034-9631 (Impreso) 
sobre el uso que le da un ejército del futuro a los mutilados, es un ejercicio que desnuda el militarismo como un absurdo. Marcos similares tienen historias como "Blitzkrieg" (una masacre ambiguamente generada por el deseo de un niño), “Tierra de nadie” o "Perros de la noche”, pero cambia completamente en otros como "Fiat mundus", un pasatiempo borgeano sobre la creación de Dios, o “Travesía”, una fantasía surrealista.

La obra de Angélica Gorodischer (1928) tiene un espacio ganado hace tiempo en la literatura argentina, pero lo que comenzó como una relación de mucha proximidad con la ciencia-ficción se convirtió en rechazo en los últimos años. La década del ochenta es de transición. Kalpa Imperial (1983-1984), un tapiz de relatos que describen un imperio ilimitado, probablemente futuro, está más cerca de la fantasía que de la ciencia-ficción. La obra fue traducida al inglés por Ursula K. Le Guin en 2003. Por fuera de esto, publicó una decena de cuentos, algunos de ellos incluidos en Las Repúblicas (1991), donde se cuentan historias ambientadas en una Argentina del futuro disgregada en pequeñas repúblicas.

La historia que inspiró el mundo de Las Repúblicas es “Llano del sol” (1982), de Elvio E. Gandolfo (1947), uno de los relatos más originales de la década. La obra de Gandolfo vinculada con la ciencia-ficción es amplia y dispersa, y va desde la narrativa y la crítica a la traducción y también a la tarea de editor. En El libro de los géneros reúne textos sobre ciencia-ficción, terror, policial y también cuentos de los tres géneros, obras que escribió a lo largo de las cuatro décadas que lleva publicando. Su crítica apareció regularmente en El Péndulo, pero su narrativa en la década es más bien escasa, y destacan en ella los cuentos “La mosca loca” (1983) y "El terrón disolvente” (1990).

Aunque fuera sólo por la cantidad de relatos que publicó durante aquellos años, la obra de Sergio Gaut vel Hartman (1947) llamaría la atención: medio centenar, en prácticamente todas las revistas del período. Pero no es sólo la cantidad, sino también la variedad temática y la búsqueda constante lo que destacan, desde el humorismo de la serie de cuentos de la Secretaría de Asuntos Estrambóticos hasta la exploración de las historias paralelas de la Argentina como "La noche de al lado” (1986). Indudablemente sus historias más conocidas son las de los “cuerpos descartables”, ambientadas en un futuro donde los cuerpos clonados se multiplican, con vida y personalidades propias, y hay cazadores dedicados a perseguirlos. Algunos de estos relatos están recopilados en el volumen que lleva el nombre de la serie, publicado en 1985.

Aunque raramente contemplada con el prisma de la ciencia-ficción, gran parte de la obra de Marcelo Cohen asume las definiciones del género. Insomnio (1986) es la historia de un personaje sin rumbo en una ciudad patagónica aislada por el ejército para que sus habitantes no emigren porque se agotó su razón de ser, el petróleo. En El oído absoluto (1989) la ciudad también funciona como marco, en este caso Lorelei, urbanización creada por un cantante popular costarricense en agradecimiento a la humanidad por su éxito. Allí todas las personas, sin distinciones sociales, pueden pasar quince días en un

\footnotetext{
(3) Revista Iberoamericana, Vol. LXXVIII, Núms. 238-239, Enero-Junio 2012, 425-439 ISSN 0034-9631 (Impreso) ISSN 2154-4794 (Electrónico)
} 
mundo utópico. Si bien la obra de Cohen del período, de una prosa intensa y evocadora, no tiene lazos significativos con el resto de la ciencia-ficción del momento (incluso fue escrita en España), debería ser tenida en cuenta en cualquier análisis del período.

No queremos terminar este repaso sin mencionar otros dos nombres cuyas obras sobresalen por sus rasgos particulares. El primer de ellos es Eduardo Abel Giménez (1954), quien publicó dos novelas (Un paseo por Camarjali, 1984, y El fondo del pozo, 1986) y un puñado de cuentos. Sus textos más largos están ambientados en un futuro distante donde una entidad llamada Centro gobierna los destinos de todos los seres humanos, pero, si bien siempre es central en las tramas, nunca se sabe a ciencia cierta qué es, quién la forma, cómo conduce los devenires. A Eduardo J. Carletti (1951) lo mencionamos como creador de Axxón, pero previamente ya había publicado varios relatos y una novela, Instante de máximo quebranto (1988). En sus historias cortas, Carletti suele trabajar con ideas y conceptos a la manera de la ciencia-ficción anglosajona, aunque no necesariamente con base científica o técnica, pero con ambientes sombríos o pesimistas. Su única novela explora el quebranto mental de un científico que es enviado a explorar la razón por la que una estrella no se convierte en supernova a pesar de que, según la ciencia conocida, así debería ser. La obra de Carletti suele ser señalada como la más cercana a la ciencia-ficción hard del período, pero esta afirmación puede desviar la atención sobre un rasgo particular: sus experimentación en temas y formas. Parte de sus cuentos están reunidos en Por media eternidad, cayendo (1991).

De este repaso hemos dejado afuera algunos nombres que publicaron textos importantes, pero que o no pueden ser enmarcados como ciencia-ficción a pesar de salir en las publicaciones reseñadas (Ana María Shua, Rogelio Ramos Signes) o escribieron y residieron un tiempo en Argentina pero su obra su publicó básicamente en Uruguay (Mario Levrero y Tarik Carson). Por último, quisiéramos dejar registrados algunos nombres de interés: Raúl Alzogaray, Santiago Oviedo, Esteban Sayegh, José Manuel López, Carlos O. Antognazzi, Graciela Parini, Eduardo L. Sánchez y José Altamirano, entre otros.

\section{CONSIDERACIONES FINALES}

Pablo Capanna ofrece una descripción de la ciencia-ficción argentina a mediados de aquella década:

Quizás el rasgo más común sea que nuestros autores no hacen ciencia-ficción a partir de la ciencia, como ocurre en países industriales donde la ciencia es una actividad socialmente prestigiosa y la tecnología impregna la vida diaria, son escritores que se han formado leyendo ciencia-ficción y en cuyo mundo espiritual importan las convenciones y los mitos del género. Decir que aquí se hace ciencia-ficción a partir de la ciencia-ficción

\footnotetext{
Revista Iberoamericana, Vol. LXXVIII, Núms. 238-239, Enero-Junio 2012, 425-439 ISSN 0034-9631 (Impreso) ISSN 2154-4794 (Electrónico)
} 
no es decir que se hace literatura de segunda mano; por el contrario, puede significar cortar camino hacia las corrientes más avanzadas del ámbito mundial. (56)

Tal vez la brecha entre la ciencia-ficción argentina y la de los países desarrollados no esté marcada tanto porque allí se escribe desde la ciencia y el entorno tecnológico sea omnipresente, este último un rasgo que puede ser sostenido para la década del ochenta. Los escritores anglosajones del género tanto como los argentinos de los ochenta se formaron leyendo ciencia-ficción, pero es cierto que en el mundo anglosajón es mucho mayor el porcentaje de escritores con formación científica que en el nuestro. De las dos culturas de Snow, está claro que la dominante en la ciencia-ficción argentina en los ochenta -y también en la actual-, es el de las humanidades.

Esta orientación, más el hecho de que las publicaciones del género no repitieran las fórmulas de las revistas anglosajonas, que repartían la ciencia-ficción y la fantasía según condiciones muy estrictas, permitieron que los escritores pudieran enlazar naturalmente su literatura con la tradición fantástica argentina, enriqueciéndola con otras formas como el surrealismo y el absurdo. Por todo esto es que resulta tan difícil de definir los rasgos dominantes de la ciencia-ficción argentina, porque no respeta las fórmulas restrictivas de la anglosajona que recién en las últimas dos décadas inició un proceso de descodificación, aproximándose y fundiéndose con la literatura general.

Pero la década del ochenta para la ciencia-ficción argentina es determinante porque por primera vez se establece un conjunto de escritores, publicaciones y lectores que manejan una serie de códigos comunes que se potencian y logran establecer de manera más o menos permanente un espacio común. Lamentablemente este fenómeno resulta difícil de estudiar porque la misma informalidad de las publicaciones y la falta de registro por parte de la academia han hecho que, a veinte años de finalizada la década, poco y nada se haya estudiado, mientras que cada vez resulta más difícil localizar aquella producción literaria. Sólo la colección de la Biblioteca Nacional contiene parte de las publicaciones del período.

Por todo esto es que nuestra intención fue preparar una cartografía de las publicaciones y autores de la ciencia-ficción argentina de los ochenta, pues en esta década de enorme dinámica es cuando el género se configuró como tal, reclamando un territorio propio, aún en los márgenes, en la literatura argentina.

\section{BiBLIOGRAFÍA}

Bell, Andrea L. y Yolanda Molina-Gavilán. "Introduction: Science Fiction in Latin America and Spain”. Cosmos latinos. Middletown: Wesleylan UP, 2005. 1-19.

Brown, J. Andrew. "Edmundo Paz Soldán and his Precursors: Borges, Diche, and the SF Canon”. Science Fiction Studies 103 34/3 (nov. 2007): 473-483.

Revista Iberoamericana, Vol. LXXVIII, Núms. 238-239,
ISSN 0034-9631 (Impreso) 
Cano, Luis C. Intermitente recurrencia: la ciencia ficción y el canon literario hispanoamericano. Buenos Aires: Corregidor, 2006.

Capanna, Pablo. Ciencia ficción: utopía y mercado. Buenos Aires: Cántaro, 2007.

"La ciencia-ficción y los argentinos”. Minotauro 10 (sept. 1985): 43-56.

Croci, Daniel. “Tesis para una nueva literatura fantástica nacional”. Nuevomundo 4 (primavera 1984): 39-41.

“Editorial”. Cuásar 1 (verano 1984): 2-3.

Fernández Delgado, Miguel Ángel. "Las ‘Sizigias y cuadraturas lunares’y el nacimiento de la ciencia-ficción en el Nuevo Mundo”. Cuásar 32 (dic. 2000): 32-40.

Ferreira, Rachel Haywood. "The First Wave: Latin American Science Fiction Discovers Its Roots”. Science Fiction Studies 103 34/3 (nov. 2007): 432-462.

Gardini, Carlos. "Entrevista”. Cuásar 3 (invierno 1984): 118-125

Gooden, Bernard y A. E. van Vogt. Lo mejor de la ciencia-ficción latinoamericana. Barcelona: Ediciones Martínez Roca, 1982.

Lafourcade, Alejandro. La revista Humor como medio opositor a la dictadura militar: tesina de grado. El Salvador: Universidad del Salvador, Facultad de Ciencias de Educación y de la Comunicación Social, Carrera de Periodismo. 2004. 5 feb. 2010 $<$ http://www.salvador.edu.ar/vrid/cpg/premio3.pdf.>

Lockhart, Darrell B., dir. Latin American Science Fiction Writers: An A-to-Z Guide. Westport: Greenwood, 2004.

Lundwall, Sam. “Aventuras en la jungla de pulpa”. El Péndulo 13 (nov. 1986): 66-78.

Molina-Gavilan, Yolanda y otros. "A Chronology of Latin-American Science Fiction, 1775-2005”. Science Fiction Studies 103 34/3 (nov. 2007): 309-431.

Molina-Gavilán, Yolanda. Ciencia ficción en español: una mitología moderna ante el cambio. Lewiston, New York: Edwin Mellen, 2002.

Pestarini, Luis. "El primer cuento argentino de ciencia-ficción”. Cuásar 30 (sept. 1998): 3-4.

Reati, Fernando. Postales del porvenir: la literatura de anticipación en la Argentina neoliberal (1985-1999). Buenos Aires: Biblos, 2006.

Revista Iberoamericana, Vol. LXXVIII, Núms. 238-239, Enero-Junio 2012, 425-439 ISSN 0034-9631 (Impreso) 
